\title{
Long Term and Short Term Effects of Perturbations in an Immune Network Model
}

\author{
Rita Maria Zorzenon dos Santos and Mauro Copelli \\ Laboratório de Física Teórica e Computacional \\ Departamento de Física, Universidade Federal de Pernambuco \\ 50670-901, Recife, PE, Brazil
}

Received on 14 May, 2003

\begin{abstract}
In this paper we review the trajectory of a model proposed by Stauffer and Weisbuch in 1992 to describe the evolution of the immune repertoire and present new results about its dynamical behavior. Ten years later this model, which is based on the ideas of the immune network as proposed by Jerne, has been able to describe a multi-connected network and could be used to reproduce immunization and aging experiments performed with mice. The immunization protocol is simulated by introducing small and large perturbations (damages), and in this work we discuss the role of both. Besides its biological implications, the physical aspects of the complex dynamics of this network is very interesting per se. In a very recent paper we studied the aging effects by using auto-correlation functions, and the results obtained apparently indicated that the small perturbations would be more important than the large ones, since their cumulative effects may change the attractor of the dynamics. However our new results indicate that both types of perturbations are important. It is the cooperative effects between both that lead to the complex behavior which allows to reproduce experimental results.
\end{abstract}

\section{Introduction}

The main task of the immune system is to protect the organism against dangerous elements: antigens (virus, bacteria, poison, cell residues, etc). Depending on the antigen the immune system may elicit different kinds of responses: the cell-mediated immune response or the humoral response. The models discussed in this paper are related to the humoral responses generated by B cells (one of the main classes of lymphocytes), which are the cells that produce the antibodies. The antibodies produced by a given population of B cells are replicas of its molecular receptor. Each molecular receptor exhibited by a given B cell population recognize different recognition sites (epitopes) of the antigen by lockkey interactions.

The clonal selection theory [1] is the most accepted theory about the B cells and was proposed by Burnet in 1959 . It states that the antigen chooses by pattern recognition the clones of B cells (population of B cell and antibodies) that will proliferate. In order to recognize any foreign (or dangerous) element the immune repertoire must be complete. According to estimates the human immune system is able to express at least the order of $10^{11}$ different receptors [1]. Due to the completeness of the repertoire, the immune system would be able to recognize and be recognized (recognizing epitopes of its own antibodies), therefore the same mechanism of recognition should work for both antibodyantigen and antibody-antibody reactions. In 1974 Jerne [2], taking into account these different mechanisms, suggested that when the antigen is presented to the organism it will ac- tivate a set of clones of B cells, leading to the production of specific antibodies; those antibodies will in turn be recognized by a second set of clones activating them, and so on. Due to the interplay of the mechanisms of activation and suppression, this chain of reactions will be finite, preventing the "information" from "percolating" through the entire system. Therefore the immune system would function as a functional network [2], with complexity comparable to the nervous system. Since its proposal, only few evidences support the existence of the immune network theory $[3,4]$, and those evidences suggest that if the network exists only $20 \%$ of the lymphocytes will be activated, while the rest of the clones will form a pool of immunocompetent lymphocytes that are able to recognize any antigen.

In what follows we define in section II a model introduced by Stauffer and Weisbuch in 1992 [5], which incorporates the main concepts of Jerne's immune network theory. In section III we review the main results obtained in the literature, focusing on both the physical $[6,7]$ and biological aspects [8], since among many other attempts to model what an immune network could be, this was the first that could reproduce the behavior of a real immune system. In section IV we present new results about the short and long time behaviors of the multi-connected network under small and large perturbations. We also discuss the results obtained about the aging effects in a very recent work [9] on the light of these new results. In our concluding remarks (section V) we address the future prospects from the physical and biological points of view. 


\section{The Model}

In 1992 Stauffer and Weisbuch (SW) [5] introduced a cellular automata model to describe the evolution of the B cell repertoire. This model was inspired in a previous one (based on a difference equation approach) introduced by de Boer, Segel and Perelson (BSP) [10] using a shape-space formalism to describe a large-scale immune network. Using a discrete shape-space formalism SW associate each point of a $d$-dimensional space to a different molecular receptor (or a clone of B cell). In this way each receptor is characterized by $d$ properties, and its neighbors in the shape space will correspond to molecular receptors that differ from it by one of these properties (according to the estimates based on a continuous approach [11], if the notion of shape space is relevant, then $d \geq 5$ ).

To each receptor they associate a three-state cellular automaton $B(\vec{r}, t)$ that will describe the concentration of the population characterized by this receptor $\vec{r}$ over the time: low $(B(\vec{r}, t)=0)$, intermediate $(B(\vec{r}, t)=1)$ and high $(B(\vec{r}, t)=2)$. Following the ideas of the immune network theory, the interactions among different populations are based on complementarity (lock-key interaction) and are defined by deterministic rules. The time evolution of the cellular automaton is based in a deterministic non-local rule: population $B(\vec{r}, t)$ at site $\vec{r}$ is influenced by the populations at site $-\vec{r}$ (its mirror image or complementary shape) and its nearest-neighbors $(-\vec{r}+\delta \vec{r})$ (representing defective lockkey interactions). The influence on the population at site $\vec{r}$ caused by its complementary populations is described by the field $h(\vec{r}, t)$ :

$$
h(\vec{r}, t)=\sum_{\vec{r}^{\prime} \in(-\vec{r}+\delta \vec{r})} B\left(\vec{r}^{\prime}, t\right)
$$

where for a given $\vec{r}$ the sum runs over the complementary shape $\vec{r}^{\prime}=-\vec{r}$ and its nearest neighbors. Due to the finite number of states of the population $B$, the maximum value of the field $h(\vec{r})$ is $h_{\max }=2(2 d+1)$. The rules are based on a window of activation for each population which is inspired in a log bell shaped proliferation function associated to the receptor cross-linking involved in the B cell activation $[6,5,10]$. There is a minimum field necessary to activate the proliferation of the receptor populations $\left(\theta_{1}\right)$, but for high doses of activation (greater than $\theta_{2}$ ) the proliferation is suppressed. The updating rule may be summarized as:

$$
B(\vec{r}, t+1)= \begin{cases}B(\vec{r}, t)+1 & \text { if } \theta_{1} \leq h(\vec{r}, t) \leq \theta_{2} \\ B(\vec{r}, t)-1 & \text { otherwise }\end{cases}
$$

but no change is made if it would lead to $B=-1$ or $B=3$. We define the densities of sites in state $i$ at time $t$ as $B_{i}(t)$ ( $i=1,2,3)$.

The initial configurations are randomly generated according to the parameter control $x$, which determines the initial concentrations: $B_{1}(0)=B_{2}(0)=x / 2$, while the remaining $L^{d}(1-x)$ sites are initiated with $B(\vec{r}, 0)=0$.

\section{Results obtained from previous studies}

The above definition of the model corresponds already to the modified version which has been proposed in Ref. [6]. SW have shown that for the original version of the model [5], there is a stable-chaotic transition on the behavior of the discrete system for $d \geq 4$, when varying the parameter $x$. As pointed out by the authors, according to the characteristics of those behaviors, none of them would be appropriate to describe the evolution of the immune repertoire.

One of us has studied how the system attains the chaotic regime, by studying the transient times and periods of the original model close to the transition [12]. It was observed that close to the transition, the system is trapped in cycles with large but finite periods. In 1995 Zorzenon dos Santos and Bernardes [6] introduced the modified version of SW model described above and have extensively studied the behavior of this model in the parameter space. Differently from SW, they obtained [6] a stable-chaotic transition for $d \geq 2$, and mapped all the behaviors on "phase" diagrams for different set of parameters, showing that there was a broad transition region between stable and chaotic behaviors.

In the following work [7] Bernardes and Zorzenon dos Santos have investigated the dynamical behavior of the model on this broad transition region. They have observed an aggregation-disaggregation dynamics, with clusters splitting and fusing along the time, as a multi-connected network. The authors have also studied the behavior of the system in the transition region when subjected to antigen presentation. The antigen presentation was simulated by flipping randomly chosen populations from the inactive state to the highly activated state, reproducing in this way the activation of the populations caused by the presence of an antigen. The results obtained by adopting the standard spread of damage procedure [13] indicate that after the antigen presentation, the perturbation first increases and then relaxes after a few time steps, indicating that some information about this perturbation is incorporated by the system. These results suggested that this model would be a good candidate to model a real immune system behavior. Zorzenon dos Santos and Bernardes then applied it [8] to reproduce immunization and aging experiments performed with mice under multiple antigen presentations $[14,15,16]$ obtained by the group of Nelson Vaz.

In the experiment of immunization [14] six mice of the same linage are subjected to the following protocol: the antigen presentations are produced by intra-venal injections of ova. The time interval between the first and second presentation is 14 days, but after that the time interval between consecutive presentations is fixed to 7 days at the same podition. Before each antigen presentation (except for the first one) they measure the amount of specific antibodies in the blood. Among many experiments reported by this group, Zorzenon dos Santos and Bernardes chose the one showing a refractory (saturation) behavior related to the immunization 
protocol [14] that could not be explained by clonal selection theory. However, the aging experiment chosen $[15,16]$ is one among others obtained by the group showing the same result: a reduction on the intensity of the response as the system ages.

In order to simulate the immunization protocol [8], for each sample they let the system evolve from its initial configuration to 1000 time steps, and associate this time step to the birth of the simulated mouse. Then random small perturbations, in size and location in the shape-space (using different time intervals between them), are produced in order to simulate the noise to which the immune system is subjected due to the environment, food ingestion, etc. By using the arbitrary scale of 1 day corresponding to 5 time steps, it is possible to simulate independent antigen presentations without over-exciting the system [7]. The immunization protocol is simulated by large perturbations (one order of magnitude greater than the small ones) being produced periodically every 7 days at the same position. The protocol was simulated for different samples (10) of young and old mice ( 8 and 24 weeks, respectively). The results could be interpreted on the light of the immune network theory, the refractory behavior being associated to the saturation of the multi-connected network in incorporating information about the perturbation, and the aging effects related to the loss of plasticity of the system.

Since there are only few evidences that support the existence of the immune network, this model played a very important role in making the connection between some experimental results that could not be explained by clonal selection theory, and the immune network theory. Moreover the theoretical results indicate that under multiple antigen presentation the saturation is due not only to the number of specific antibodies produced (as shown in the ELISA experiments) but also the ability to incorporate information by including new populations in the network. Since the number of populations belonging to the network does not change significantly during the time evolution, in order to add information, part of the information already present in the network should be discarded. Therefore the profile of expressed antibodies would change after each antigen presentation. This is an aspect that comes out of the theoretical results and should be investigated by the experimentalists when the appropriated tools become available. The techniques currently available do not allow to identify the difference among the specific antibodies. That would be a requirement to verify whether they change after multiple presentations as suggested by the model. However, there are some experimental evidences that it may happen [17].

Once the complex multi-connected network generated by the model can be used to reproduce the behavior of a real immune system, it becomes interesting to investigate its dynamical properties from the physical point of view. This investigation will allow to learn about the dynamics of a (real) complex system operating out of equilibrium. The characterization of such behavior started with the work of Bernardes and Zorzenon dos Santos [18], who analyzed the distribution of cluster sizes and the distribution of the permanence time (i.e. the time interval during which each popula- tion remains activated or belong to the multi-connected network). They found a characteristic cluster size and a power law behavior for the distribution of permanence times. The characteristic cluster size is associated to the loss of plasticity while the power law distribution indicate that the populations belonging to the network have no typical permanence time, reflecting the fact the dynamical memory is generated by incorporating information about the different antigens presented to the system.

In a very recent work in collaboration with D. Stariolo [9] we have used the auto-correlation functions (a common tool to study aging effects in e.g. glassy systems) in order to analyze the similarities between the aging effects in the multi-connected network and in glassy systems. While in glassy systems the aging effects results from the loss of plasticity generated by frustration mechanisms, in the network these effects are caused by multiple perturbations, since in order to incorporate information the system has to adapt to (or satisfy) the mechanisms of activation and suppression of the dynamics. The usual procedure adopted to study glassy systems consists in taking a "picture" of the system at time $t_{w}$ and calculating the number of sites that do not change during the following time steps. In this sense the auto-correlation function is equal to $C_{\text {tot }}\left(t, t_{w}\right)=1-h d\left(t, t_{w}\right)=1-H D\left(t, t_{w}\right) / N$, where $h d\left(t, t_{w}\right)\left[H D\left(t, t_{w}\right)\right]$ is the normalized (non-normalized) Hamming distance between the configurations at times $t$ and $t_{w}$. According to the results obtained, the system without any perturbation is driven to a long period attractor after a long transient time $\left(10^{4}-10^{5}\right.$ time steps). All the biologically relevant effects are observed in the transient time of the system. In the purely deterministic case, the transient is simply the time it takes for the system to reach the attractor $\left(\sim 10^{4}\right.$ time steps). When subjected to random small perturbations, however, the very notion of a transient becomes fuzzy: results in Ref. [9] show that the system approaches a cycle, but is deflected from it by the small perturbations after $\sim 10^{3}-10^{4}$ time steps. Therefore, the small perturbations will cause the system to change attractors from time to time due to their cumulative effects. This is reflected in the decrease of the auto-correlation functions, as can be seen Fig. 1, where the system leaves the cycle it had approached until $t_{w}=10^{5}$ time steps (these results are discussed in detail in Ref. [9]). The analogue observed in glassy systems corresponds to changes of the system to different minima of the energy landscape during the relaxation time. Contrary to one's initial intuition that the large perturbations would accelerate the de-correlation process, the changes induced by large perturbations do not lead to this effect. Curiously, the large perturbations alone (which correspond to the immunizations) lead to a much weaker (slower) de-correlation: this is due to the fact that they are produced always at the same sites in shape space. Small perturbations can be more easily absorbed by the system than the large ones since they involve only local changes. The results from the autocorrelation functions therefore indicate that the system first approaches a cycle (phase space compression), but after some time the small perturbations eventually deflect it from the trajectory to its "natural" attractor [9]. 

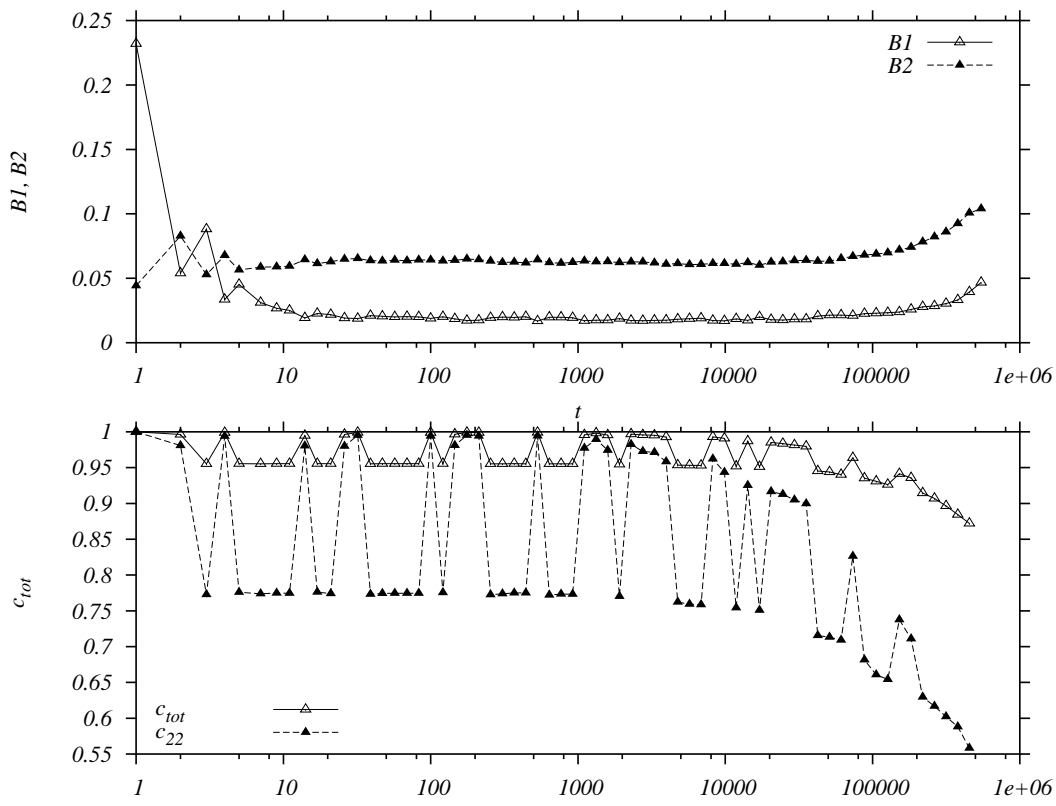

Figure 1. Densities vs. time (upper panel) and correlations vs. $t-t_{w}{ }^{t-t_{w}}$ (lower panel) for a system with small perturbations, $L=50, x=0.26$ and $t_{w}=10^{5} . C_{t o t}$ corresponds to the autocorrelation function defined in the text. $C_{22}$ measures the autocorrelation only in the subspace of sites with $B\left(\vec{r}, t_{w}\right)=2$.

\section{Results}

This scenario provides a possible explanation for the results regarding the spread of damage in the system, which managed to reproduce the experimental data from immunization experiments with mice [8]. Fig. 2 shows the time evolution of the Hamming distance between a system which undergoes only small perturbations, and its copy, which undergoes the same small perturbations and the large periodic immunizations. All curves saturate after some time, just like the ELISA measurements [8]. More important, however, is the fact that the value of the saturation depends on the age at which immunization started. As the age increases, the saturation value decreases, a result which was also observed in the mice and is interpreted as a sign of loss of plasticity with age [8]. This might be related to the fact that the system is in its transient, while trying to reach its long-period attractor. The "older" the system is, the less configurations available there are.

If we now repeat the computer experiment of immunization (with small and large perturbations) for ages greater than $\sim 10^{3}$ time steps, we obtain a different result: for older systems the stationary Hamming distance may increase, as shown in the rightmost curve of Fig. 2. We argue that the cumulative effects of the small perturbations could be responsible for the change of behavior in the rightmost curve of Fig. 2: for sufficiently old systems, the "noise" of the small perturbations become more important than earlier in the transient, and the monotonicity of the stationary value of the HD with the age at which immunization starts, no longer holds. This result is summarized in Fig. 3. It is interesting to point out that this breakdown of the refractoriness induced by age, takes place on time scales which are comparable (typically larger than) the life time of the mice.

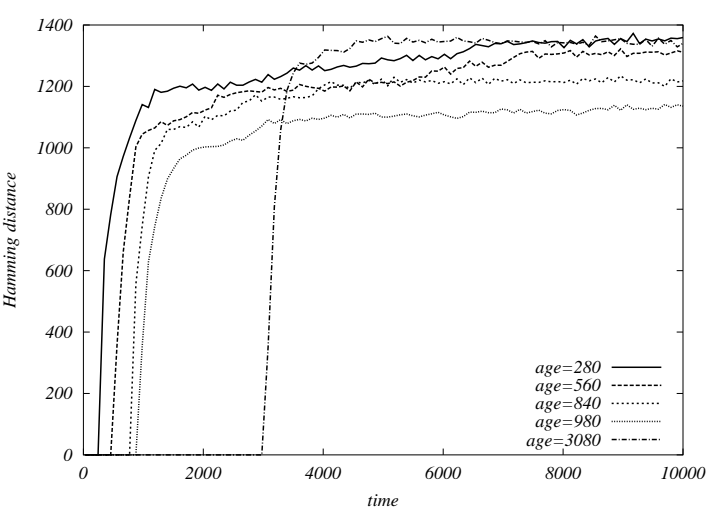

Figure 2. Mean Hamming distance vs. time for the complete immunization protocol adopted in Ref. [8] and for different ages. Average over 10 runs. Standard deviations are not shown for the sake of clarity.

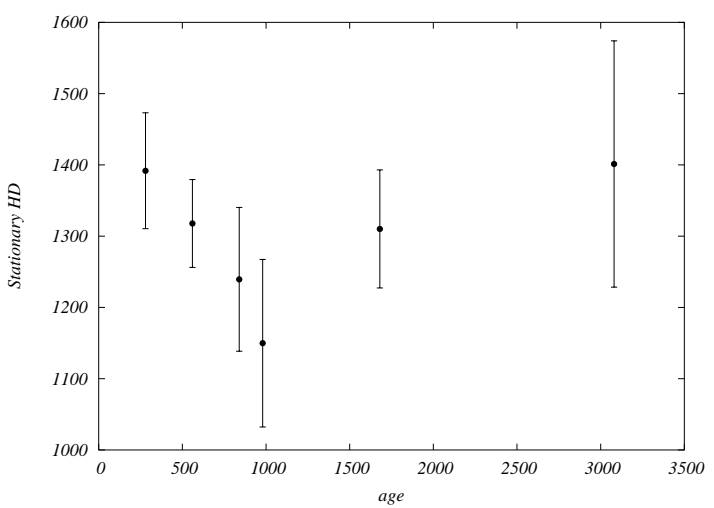

Figure 3. Stationary Hamming distance vs. age at which the immunizations start. Bars correspond to standard errors (average over 10 runs). 
The large perturbations, on the other hand, propagate to the entire system and some of the active populations generated during the antigen presentation will remain as part of the network. This mechanism depends on the plasticity of the system or, in other words, will be controlled by the characteristic cluster size of the system [18]. When we combine small and large perturbations, the system is driven to a cycle that incorporates information about the antigen, but will eventually be deviated from that trajectory as soon as the cumulative effects of the small perturbations dominate the dynamics [9]. In order to observe the instantaneous effects of both types of perturbations, we have analyzed the normalized Hamming distance between the same system at two consecutive time steps, focusing only on the subspace of sites with $B(\vec{r}, t)=2$. This quantity is denoted by $H D_{22}(t, t+1)$ and referred to simply as "instantaneous Hamming distance". It is plotted in Fig. 4 as a function of time for different situations.

We start the analysis of Fig. 4 by noticing the behavior for the purely deterministic case (lower curve): the plot indicates that typically $2.6 \%$ of the active network (sites with $B(\vec{r}, t)=2)$ changes at each time step. The end of the transient is clearly seen at $t \sim 15000$ time steps (for this particular realization): the curve then becomes periodic, with a very long period. Note that the periodicity of the dynamics can be confirmed by checking that the $C_{t o t}\left(t_{w}, t\right)=1$ for some $t>t_{w}$ and for sufficiently large $t_{w}$ (see Fig. 1 and Ref. [9]). To illustrate the periodicity of $H D_{22}(t, t+1)$, we plot in Fig. 5 the return map of the time series from $t=20000$ to $t=25000$ time steps (upper plot). The extremely long period might lead one to suspect, based on the return map, that the system might be chaotic. This suspicion is ruled out when one observes the return map from $t=25000$ to $t=30000$ time steps (lower plot). We challenge the reader to find a discrepancy between the two return maps.

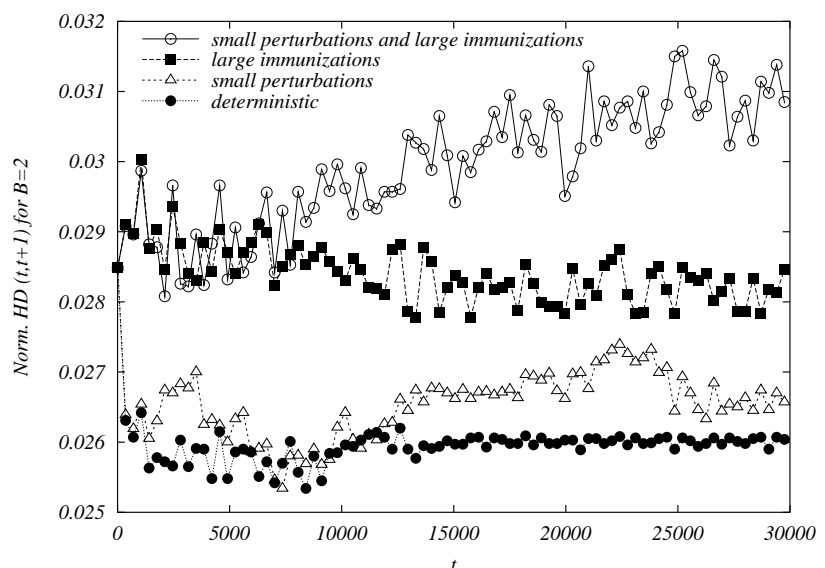

Figure 4. Normalized Hamming distance between configurations at times $t$ and $t+1$, taking into account only activated $(B=2)$ sites.
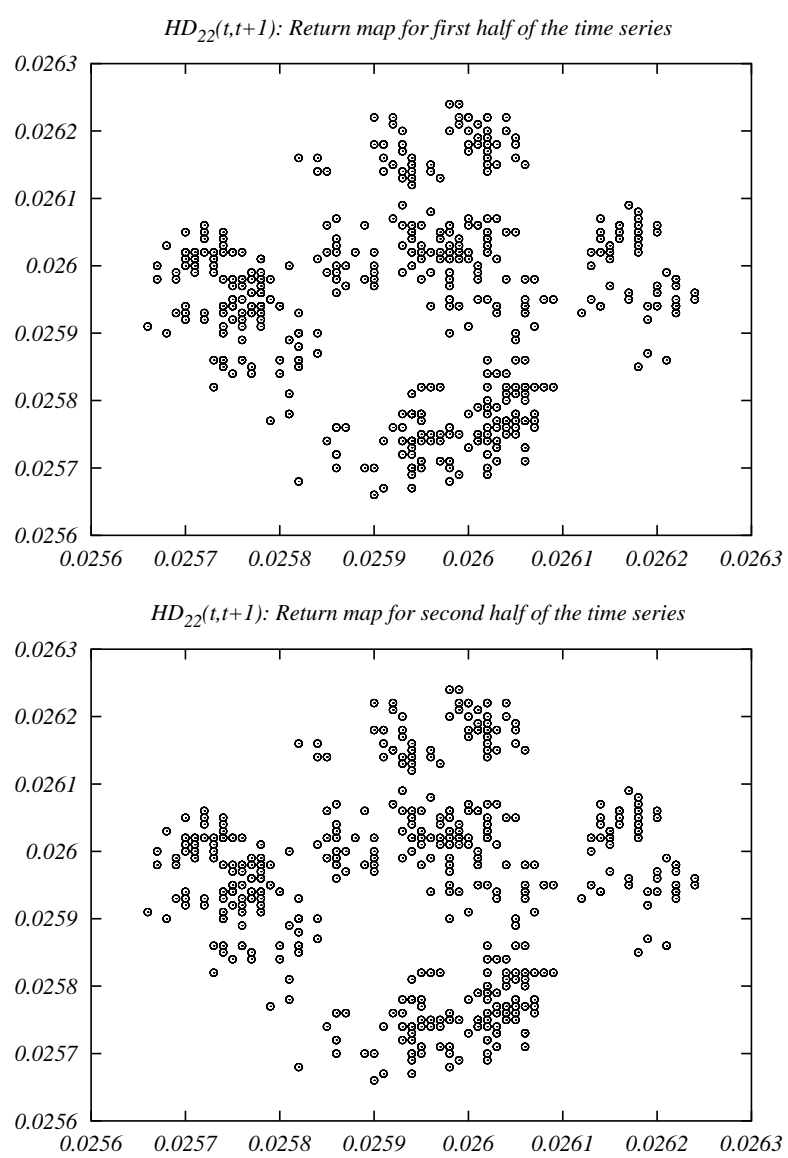

Figure 5. Top panel: return map of $H D_{22}(t, t+1)$ from $t=20000$ to $t=25000$. Bottom panel: return map of $H D_{22}(t, t+1)$ from $t=25001$ to $t=30000$

Returning to Fig. 4, notice that the size of the changes induced by the small perturbations changes near the end of the transient time (second curve from bottom to top). While the perturbed system no longer attains the cycle, it can still feel the effects of the attractor: when close to the cycle, the small perturbations induce larger changes in the active network at each time step. If subjected only to the large immunizations (third curve from bottom to top), the instantaneous Hamming distance take typically larger values. This might suggest a contradiction with the results in Ref. [9], according to which the system only with large immunizations de-correlates slower. But the contradiction is only apparent: $H D\left(t_{w}, t\right)$ measures a long term effect, which is dominated by the small perturbations. Fig. 4 , on the other hand, is a measure of the instantaneous change occurring in the system, i.e. a short term effect. Finally, one can combine both types of perturbation (upper curve), the result being even larger instantaneous changes.

Another aspect of Fig. 4 that should be noted is an interesting interplay between the perturbations. In what follows, we refer to the typical values of the four curves in Fig. 4 (for large $t$ ), from bottom to top, as $d$ ("deterministic"), $s$ ("small"), $l$ ("large") and $l s$ ("large and small") therefore $d<s<l<l s$, as discussed above. The fact that $l s-s>l-d$ indicates that the effect of the large 
immunizations (i.e. the instantaneous change it induces) is boosted by the presence of the small perturbations. The reverse is obviously also true: the previous inequality implies $l s-l>s-d$, indicating that the small perturbations induce larger changes in the network if the large immunizations are present. This signals a cooperative interaction between two kinds of perturbations which are significantly different in nature, leading to the necessary complexity of behavior which allows the model to reproduce experiments performed with real immune systems.

\section{Concluding Remarks}

In this paper we have reviewed the main results from the biological and physical point of view of a cellular automata model introduced by SW in 1992. Since it incorporates the main features of the immune network theory and models the functioning of a multi-connected network, it emerged as a candidate to reproduce the basic dynamics of what the real immune network could be. The model was then used to model real experiments performed with mice about immunization and aging. The immunization experiments are simulated by producing small and large perturbations or damages. In this paper we complement the results of a very recent work [9] with the study of the role of each kind of perturbation. The results obtained in the previous work, using auto-correlation functions, apparently suggested that the small perturbations would be more important than the large ones from the dynamical point of view, since their cumulative effects could change the attractor of the dynamics after a few thousand time steps. However this study was focusing in the long term behavior of the system. Here we have studied its short term behavior and shown that both types of perturbation are important: it is their cooperative effects that generate the necessary complexity which allowed to reproduce experiments performed with real immune systems.

Despite the understanding of the dynamics we have achieved up to this point, there are still some points that should be investigated: what happens when changing the frequency of presentation of the small perturbations for a fixed value of the large damages, and how these results will change when varying the large damage size? This study (already in progress) will draw a scenario that would allow us to try to apply this model to reproduce other experimental results concerning, for instance, cross-reactivity mechanisms observed in immunization protocols, and tolerance.
The authors acknowledge support from $\mathrm{CNPq}$, PropesqUFPE (Programa Enxoval) and PRONEX.

\section{References}

[1] C. A. Janeway, P. Traver, M. Walport, and J. Capra, Immunobiology: the immune system in health and disease, Elsevier Science - Garland Publishing, NY (1999), 4th edition.

[2] N. K. Jerne, Ann. Immuno. (Inst. Pasteur) 125 C , 373 (1974).

[3] A. Coutinho, Immunol. Rev. 110, 63 (1989).

[4] D. Holmberg, Ånderson, L. Carlsson, and S. Forsgren, Immunol. Rev. 110, 84 (1989).

[5] D. Stauffer and G. Weisbuch, Physica A 180, 42 (1992).

[6] R. M. Zorzenon dos Santos and A. T. Bernardes, Physica A 219, 1 (1995).

[7] A. T. Bernardes and R. M. Zorzenon dos Santos, J. Theor. Biol. 186, 173 (1997).

[8] R. M. Zorzenon dos Santos and A. T. Bernardes, Phys. Rev. Lett. 81, 3034 (1998).

[9] M. Copelli, R. M. Zorzenon dos Santos, and D. A. Stariolo, Eur. Phys. J. B 34, 119 (2003).

[10] R. J. De Boer, L. A. Segel, and A. S. Perelson, J. Theor. Biol. 155, 295 (1992).

[11] A. S. Perelson and G. F. Oster, J. Theor. Biol. 81, 645 (1979).

[12] R. M. Zorzenon dos Santos, Physica A196, 12 (1993).

[13] N. Jan and L. de Arcangelis, Annual Reviews of Computational Physics I, ed. by D. Stauffer, World Scientific, 1-12 (1994).

[14] B. A. Verdolin, MSc. Thesis, Departamento de Bioquímica e Imunologia, Instituto de Ciências Biológicas, UFMG, Belo Horizonte (1997); A. M. C. Faria, S. M. Ficker, E. Speziali, J. S. Menezes, B. Stransky, B. A. Verdolin, W. M. Lahmann, V. S. Rodrigues, and N. M. Vaz, Braz. J. Med. Biol. Res. 31, 35 (1998); N. M. Vaz, A. M. C. Faria, B. A. Verdolin, and C. R. Carvalho, Scand. J. Immunol. 46, 225 (1997).

[15] A. M. C. Faria, S. M. Ficker, E. Speziali, J. S. Menezes, B. Stransky, V. S. Rodrigues, and N. M. Vaz, Mech. Ageing Dev. 102, 67 (1998).

[16] W. M. Lahmann, J. S. Menezes, B. A. Verdolin, and N. M. Vaz, Braz. J. Med. Biol. Res. 25813 (1992).

[17] G. Teixeira, private communication.

[18] A. T. Bernardes and R. M. Zorzenon dos Santos, Int. J. Mod. Phys. C 12, 1 (2001). 